\title{
LA APLICACIÓN DE LOS MUNDOS INMERSIVOS PARA LA RESOLUCIÓN DE PROBLEMAS ASOCIADOS A CASOS REALES EN EL MUNDO DE LA INGENIERÍA AGRONÓMICA, DE MONTES Y DE LA ENOLOGÍA
}

\author{
APPLIYING INMERSIVE WORLDS FOR SOLVING REAL PROBLEMS OF AGRONOMIC AND FOREST \\ ENGINEERING AND ENOLOGY
}

\author{
E.V. Taguas*, E. Fernández-Ahumada, \\ L. Ortiz-Medina, P. Sánchez-Zamora, \\ T. De Haro-Giménez, S. Andicoberry, \\ P. Lara-Vélez, F. Montes-Tubío, \\ F. Mérida, L. Moya, R. Gallardo-Cobos \\ evtaguas@uco.es \\ Universidad de Córdoba
}

Received: 28/06/2017 Accepted: 20/02/2018

\begin{abstract}
Resumen
El Plan de Innovación Docente 2016-2017 de la Universidad de Córdoba planteaba en sus líneas prioritarias de proyectos el trabajo por competencias, las actividades académicamente dirigidas y la transferencia del conocimiento teórico a la práctica. En este contexto, se planteó desarrollar una serie de casos prácticos en un espacio virtual adaptado a las características de los mismos donde, a través de juegos de rol (en los que participaran no sólo los estudiantes sino profesionales del sector) los estudiantes organizados en grupos tendrían que resolver problemas y/o plantear y analizar sus posibles soluciones. Se presenta en el siguiente trabajo un proyecto colectivo donde diferentes escenarios prácticos y agentes involucrados en los temarios de asignaturas como Proyectos y Comercialización y Regulación de Mercados Agrarios comunes al Grado de Ingeniería Agroalimentaria y del Medio Rural, Ingeniería. Forestal y Enología fueron representados. En el primer caso, se propuso la elaboración y presentación de una licitación pública y en el segundo caso, la simulación de los actores de una subasta pública en una lonja.

Entre los resultados del proyecto, se destacan los vídeos protagonizados por los alumnos reproduciendo las entrevistas reales y negociaciones de los agentes de la lonja o en el caso de la licitación, la preparación de los pliegos de una licitación por parte de los técnicos de un ayuntamiento, la elaboración de las plicas por parte de empresas licitadoras y la evaluación de las mismas por parte de la mesa de contratación. Además, los resultados de las encuestas a los estudiantes pusieron de manifiesto la importancia de este tipo de proyectos para mejorar la adquisición de conocimiento práctico de las asignaturas así como competencias de comportamiento y particularmente de tipo creativo.
\end{abstract}

Palabras clave: mundos inmersivos; juego de rol; avatares; licitación; subasta pública.

\begin{abstract}
The Teaching Innovation Plan 2016-2017 of the University of Cordoba proposed in its priority lines of projects the work by competences, the academically directed activities and the transfer of theoretical knowledge to the practice. In this context, the development of a series of practical cases in a virtual space or role plays were proposed in which not only students but professionals of the sector participated. The students, organized in groups, had to solve problems and / or raise and analyze their possible solutions. We presented in the following work a collective project where different practical scenarios and agents involved in the subjects "Projects" and "Marketing and Regulation of Agrarian Markets" of the Degrees of Agri-food Engineering and Rural Environment, Forest Engineering and Oenology were represented. The first case was the preparation and the presentation of a public contract whereas in the second case, the simulation of the actors of a public auction was played.

Among the results of the project, it is worth highlighting the videos played by the students reproducing the actual interviews and negotiations of the agents of the auction or in the case of the tender, the preparation of the bidding documents by the technicians of a town hall, the offers of the bidding companies and the evaluation of the same by the contracting table. In addition, the results of the student surveys showed the importance of this type of projects to improve the acquisition of practical knowledge of the subjects as well as behavioural competences and, particularly, of a creative type.
\end{abstract}

Keywords: immersive world; game of role; avatars; public projects; auction.

\section{INTRODUCCIÓN}

El Espacio Europeo de Educación Superior (EEES) promueve mejoras en las metodologías de enseñanza a través de las cuales los estudiantes, especialmente los estudiantes de ingeniería y enseñanzas técnicas debido a su perfil profesional, incorporen competencias básicas asociadas a la resolución de problemas reales (Taguas et al, 2014). El aprendizaje basado en la resolución de problemas permite desarrollar su pensamiento crítico y mejorar sus habilidades sociales, debido a que sus conclusiones tienen que ser compartidas o defendidas. Al mismo tiempo, aspectos como la creatividad, la autonomía, el aprendizaje permanente y la capacidad de análisis se fortalecen cuando los estudiantes trabajan en escenarios comunes o casos reales que reflejan su futuro como profesionales (Schaf et al., 2009; Rodríguez-Donaire y Amante, 2012; Redel et al., 2014). En esta línea, son numerosos los autores que defienden que la estrategia principal de la educación moderna debe centrarse en la actividad independiente del alumnado, la organización de entornos de autoaprendizaje y la formación experimental y práctica, donde los estudiantes tienen la opción de llevar a cabo acciones y tener la iniciativa. De esta forma, se hace necesario el uso de métodos interactivos de formación, que fomenten el interés por la profesión; promuevan la adquisición eficiente de conocimiento; formen patrones de conducta; proporcionen una alta motivación y conciencia de equipo; y lo más importante, contribuyan a la adquisición de las complejas competencias que deben tener los profesionales del futuro. 
Dentro de la metodología interactiva existen diversas estrategias para activar la participación del alumnado. Entre otros, los juegos de rol, al hacer experimentar determinadas situaciones, permiten practicar y desarrollar habilidades, trabajar de manera colaborativa en pequeños grupos, y observar y reflexionar sobre actitudes y valores que en el método convencional expositivo difícilmente podrían ponerse en acción. Por otro lado, herramientas basadas en las nuevas tecnologías de la información y la comunicación abren un abanico de posibilidades para la implementación de metodologías interactivas. En concreto, la realidad virtual y los mundos inmersivos, con ambientes artificialmente generados por ordenador donde los usuarios interactúan a través de un individuo figurativo que los representa en dicho ambiente (su “avatar"), han sido señalados como los elementos tecnológicos de principios del siglo XXI que cambiarán más dramáticamente el modo en que vivimos, particularmente en las áreas educativas, de desarrollo de productos y de entretenimiento (Schmorrow, 2009)

La conjunción de estrategias de juegos de rol y los mundos virtuales inmersivos constituye una herramienta con enorme potencial para la recreación de escenarios donde los estudiantes pueden asumir temporalmente identidades y tareas sin incurrir en consecuencias en el mundo real. Así, a partir de un software de dominio multiusuario, cada participante puede estar presente en el entorno tridimensional creado a través de su representación gráfica "avatar" que interactúa y emula una situación real (Baker et al., 2009). El uso de "avatares" favorece la interacción por su carácter lúdico y distendido, comúnmente asociado a videojuegos o a animación. De esta manera, en este trabajo se planteó que los estudiantes utilizaran un espacio del mundo virtual adaptado al escenario de una situación profesional elegida, para interaccionar entre ellos o con profesionales externos representando el papel del agente externo dentro de las negociaciones de una lonja de productos agroalimentarios o en el marco de una licitación pública.

\section{OBJETIVOS}

El objetivo principal de este trabajo fue el diseño de juegos de rol en el contexto de distintas asignaturas comunes a las titulaciones del Grado en Ingeniería Agronómica y del Medio Rural, Ingeniería. Forestal y Enología asociados a un espacio virtual inmersivo adaptado para tal fin para que los estudiantes empatizaran, reflexionaran e incorporaran competencias dirigidas a la resolución de casos prácticos reales.

Este objetivo principal puede dividirse en los siguientes objetivos específicos:

1) Diseño de experiencias educativas donde se exploren los puntos críticos de los casos para el análisis de decisiones y su impacto.

2) Aplicación de los mundos inmersivos para la escenificación de las dinámicas y la mejora del aprendizaje.

\section{METODOLOGÍA}

Se diseñaron 5 paquetes de trabajo o capítulos vinculados a los objetivos de la propuesta para cada asignatura, con excepción del quinto que es global: 1) Diseño de los casos (juegos de rol) vinculados a las asignaturas; 2) Adaptación de los escenarios de mundos inmersivos a las dinámicas propuestas; 3) Ejecución y preparación de vídeos que puedan mostrar la experiencia y hacerla útil de un modo sencillo y funcional para otros cursos; 4) Evaluación (Feedback) de la resolución de los casos; 5) Análisis global sobre las lecciones aprendidas (Anexo; Figura 1). A continuación se presentan los paquetes de trabajo y el conjunto de actividades ligadas a los mismos. Además se adjunta el cronograma seguido.

1) Diseño de los casos a resolver que se presentan a continuación (Objetivo 1):

- Proceso de licitación pública. Asignatura: Proyectos, troncal, $3^{\circ}$ Grado de Ingeniería Agronómica y del Medio Rural (incluida también en $4^{\circ}$ del Grado de I. Forestal).

- Compra de aprovisionamiento en la subasta de una lonja (evaluación de precios, calidades, vida útil, tipo de envasado, etc.). Asignatura: Comercialización y Regulación de Mercados Agrarios, obligatoria, $3^{\circ}$ Grado de Ingeniería Agroalimentaria y del Medio Rural y $3^{\circ}$ Grado de Enología.

En el contexto de cada asignatura, se planteó la recreación de un caso real. Se definieron los agentes implicados para la asignación de roles, se plantearon los hitos y los puntos críticos de los distintos escenarios para el análisis de decisiones, se exploró la legislación aplicable para los informes que se pidieron a los alumnos en cada caso y se preparó un guion de trabajo para introducirlo en las sesiones prácticas de las asignaturas.

2) Adaptación del espacio virtual a los casos propuestos (Objetivos 1 y 2). Se utilizó las herramientas de OPEN SIM para la construcción de escenarios adecuados a los casos propuestos asignando diferentes estancias y de acuerdo a las necesidades descritas en la actividad 1; uso de multimedia, modelos, etc.

3) Realización de los juegos de rol en la plataforma virtual (Objetivo 2). Generación de grabaciones y scripts para la elaboración de material transferible. Para ello, los participantes entraron en el mundo virtual y llevaron a cabo la actividad asociada al Objetivo 1, de la cual se obtuvieron vídeos que fueron evaluados o incluso premiados, como se desarrollará en la sección de resultados.

4) Medidas del impacto de la experiencia a partir de cuestionarios de análisis a alumnos y profesores vinculados a las asignaturas sobre aprendizaje realizada por la UCO (Objetivos 1 y 2). En las sesiones de trabajo se recogió la información relativa a la curva de aprendizaje, la organización, ejecución y resultados de las sesiones de trabajo para valorar las experiencias.

5) Discusión de las lecciones aprendidas, fortalezas y debilidades de la experiencia. 


\section{DESCRIPCIÓN}

Aunque inicialmente la experiencia se había planificado también para las asignaturas Nuevas Disciplinas en Gestión de Proyectos (Optativa Máster Ingeniero Agrónomo) y Taller de Empresas (Grados de I. Agroalimentario y del Medio Rural y Grado de I. Forestal), el reducido número de alumnos en el primer caso y los problemas técnicos solapados por el uso de los mundos inmersivos en las asignaturas del segundo cuatrimestre, que se desarrollarán en la discusión impidieron su aplicación. De esta forma y como se ha reflejado en la Figura 1 del Anexo, el proyecto se concentró en dos asignaturas troncales de entre 80 y 90 alumnos impartidas en el primer y segundo cuatrimestre respectivamente. Se presentan las experiencias en detalle a continuación.

\subsection{PROYECTOS}

El diseño de la práctica se entroncó en los objetivos del correspondiente a Contratación de Proyectos (tema 5), en el cual se desarrollaban las pecualiaridades de la contratación pública y el proceso de licitación. De acuerdo a los paquetes de trabajo se desarrollaton las siguientes etapas:

1) El programa del curso se estructuró de la siguiente forma: i) 4 h de clase magistral sobre el tema con una dinámica de grupo sobre contratación privada; ii) una sesión práctica donde los estudiantes debían leer e interpretar los pliegos de una licitación pública real correspondiente a la reparación de una camino real por parte de la empresa Tragsa y donde los alumnos tenían que preparar la oferta y el calendario; y iii) la sesión de mundos inmersivos donde se proporcionaba un espacio virtual con una antigua nave donde se presentaba una licitación pública por parte de un ayuntamiento para transformarla en un centro cultural de usos múltiples. Se puede ver el esquema explicativo en la Figura 2 del Anexo.

2) En relación a la adaptación de los mundos virtuales para la práctica, además de la nave (Figura 2; Anexo) y espacios de tipo "oficina" y "cafetería", se crearon distintos usuarios para los distintos tipos de agentes: técnicos de ayuntamiento, empresas licitadoras y miembros de la mesa de contratación (Anexo, Figura 3). Como la asignatura contaba con 86 matriculados, la práctica se organizó en 4 grupos de práctica de entre 20 y 25 alumnos. Como puede verse en la Figura 3, cada estudiante tuvo su propio avatar.

3) En la sesión práctica, se presentaron los hitos temporales de trabajo y el material que se proporcionaba para llevarla a cabo. En la Figura 3 puede verse cómo tras la organización de los grupos, los estudiantes tuvieron que caracterizar su avatar y familiarizarse con la nave de la licitación y los escenarios disponibles para representar el agente asignado. Además, cada grupo se reunió con los profesores para clarificar la situación a representar (que tenía que presentarse como vídeo) y la documentación a entregar (ver Tabla 4). La fecha límite de entrega a través de Moodle fue el día 15 de mayo y el material de apoyo (ver Figura 4 del Anexo), fueron: i) un manual de manejo del avatar, ii) el guion de la práctica; iii) la presentación del Tema y iv) las propias explicaciones de los profesores a lo largo de las sesiones teórico-prácticas y tutorías individuales.

Tabla 1. Descripción de las entregas correspondientes a la práctica de acuerdo al grupo.
\begin{tabular}{|l|l|}
\hline Agente & Entregas \\
\hline $\begin{array}{l}\text { Técnicos del } \\
\text { Ayuntamiento }\end{array}$ & $\begin{array}{l}\text { Pliegos de la licitación. Vídeo escenificando alguna } \\
\text { situación asociada }\end{array}$ \\
\hline $\begin{array}{l}\text { Empresa } \\
\text { licitadora }\end{array}$ & $\begin{array}{l}\text { Documentación técnica, económica y administrativa - } \\
\text { preparación de las plicas } \\
\text { Vídeo escenificando alguna situación asociada }\end{array}$ \\
\hline $\begin{array}{l}\text { Mesa de de constitución, acta de calificación y acta de } \\
\text { Contratación }\end{array}$ & $\begin{array}{l}\text { Acta de condión } \\
\text { adjudicación de la licitación. } \\
\text { Vídeo escenificando alguna situación asociada }\end{array}$ \\
\hline
\end{tabular}

4) El día 19 de mayo, tras haber evaluado las entregas de los estudiantes y haberlas puesto en común con el gerente de Aguas de Córdoba, D. Luis Moya, quien además había proporcionado documentación e información para el diseño de la práctica, se procedió a lo siguiente: i) proporcionar un feedback a los estudiantes del resultado de sus trabajos en términos de errores y propuestas de mejora (ver Figuras 5 y 6 del Anexo); ii) la entrega del primer premio PLICA-PLICA a los estudiantes con el mejor trabajo tanto desde el punto de vista documental como de escenificación (ver Figura 7 del Anexo). En relación a la difusión, la foto de los ganadores fue "twitteada" a la cuenta de la ETSIAM el día 19 de mayo-de 2017.

\subsection{COMERCIALIZACIÓN Y REGULACIÓN DE MERCADOS AGRARIOS}

En esta asignatura la escenificación de los mundos inmersivos se incluyó como práctica del tema 6 de la asignatura, referente a los Centros de Contratación o "mercados" de productos agrarios. El programa de dicho tema constaba de: i) 2 h de clase magistral; ii) $2 \mathrm{~h}$ de sesión sobre mundos inmersivos impartidas a cada uno de los grupos reducidos creados ad hoc para esta práctica; iii) una visita con salida al mercado mayorista (lonja) de la ciudad de Córdoba (MERCACÓRDOBA) de 4 h de duración; y finalmente iv) una sesión de trabajo colaborativo por pequeños grupos de alumnos para elaborar el trabajo requerido en la práctica. 
Las fases en las que se abordó el programa se detallan a continuación:

1) En relación a la clase teórica magistral celebrada el día 17 de octubre de 2016, los alumnos recibieron formación sobre los mercados centrales mayoristas, su evolución y perspectiva, así como sobre la política española de mercado de destino (MERCASA y su red de "mercas").

2) Paralelamente, los miembros del proyecto crearon edificios de oficinas y espacios de negociación en los mundos virtuales para llevar a cabo la parte práctica. Como la asignatura contaba con 109 matriculados, de los que 75 de ellos tenían que hacer obligatoriamente la parte práctica, ésta se organizó en 4 grupos de unos 20 alumnos.

3) Las dos sesiones prácticas (1 sesión por cada 40 alumnos) sobre mundos inmersivos se realizaron en sendas aulas informáticas del Campus de Rabanales las fechas 17 y 19 de octubre de 2016. Durante ambas sesiones se presentaron los objetivos de la práctica que consistían esencialmente en simular el tipo de contratación que se lleva a cabo en los mercados de la red MERCASA. Además, en esta práctica se introdujo a los alumnos en el manejo del software libre utilizado para los mundos inmersivos, se asignaron los avatares tanto para compradores como para vendedores, se familiarizaron con las distintas opciones que proporciona la herramienta y se entrenaron para poder abordar los objetivos de la práctica propuesta.

4) La visita a MERCACÓRDOBA se realizó el día 26 de octubre de 2016. En el contexto de la visita, los alumnos recibieron $1 \mathrm{~h}$ de formación práctica sobre el funcionamiento real del mercado y, para realizar su práctica, cada grupo de alumnos seleccionó libremente uno de los productos comercializados en el mercado (fruta u hortaliza), observaron y preguntaron a mayoristas y minoristas sobre su comportamiento en sus operaciones de compra-venta bilaterales.

5) La sesión de trabajo colaborativo la realizaron los alumnos en sus diferentes grupos de trabajo a partir del 26 de octubre y hasta el 5 de noviembre. De forma sintética, este trabajo colaborativo consistió en la simulación para la fijación del precio de una fruta u hortaliza (elegida por el subgrupo de alumnos) en base a los precios reales que obtuvieron en su visita a MERCACÓRDOBA. Para dicha simulación, el subgrupo de alumnos con rol asignado de vendedor debía partir del precio máximo del producto real seleccionado (fruta u hortaliza) de los ofrecidos por los mayoristas, y el subgrupo de alumnos con rol asignado de comprador debía arrancar del precio mínimo de ese mismo producto (precio inicial del minorista). La negociación y su resultado final con la fijación del precio de compra-venta, se tenía que reflejar para su evaluación, a través del uso del software libre, en un vídeo con audio de un minuto máximo de duración.

6) El día 7 de noviembre de 2016, se procedió a la evaluación de esta práctica para lo que se invitó a todos los participantes del proyecto (alumnos y profesores), y también a D. Francisco Mérida, como agente externo para evaluar el resultado del trabajo de los estudiantes. Además se organizó una sesión con los estudiantes para comentar posibles mejoras en las estrategias de negociación.

El día 5 de junio el equipo del proyecto se reunió para la preparación y discusión de las conclusiones finales del proyecto de acuerdo a la información recopilada en ambas asignaturas y para completar el trabajo.

\section{RESULTADOS OBTENIDOS}

En primer lugar se ha consolidado una metodología de trabajo para la resolución de problemas en escenarios profesionales asociados a las titulaciones de acuerdo a los Objetivos 1 y 2 . En segundo lugar se ha elaborado un material digital docente que permite visualizar el proceso de aprendizaje para su uso en cursos siguientes así como una pequeña guía de lecciones aprendidas sobre los casos propuestos (Objetivo 2).

Además, a través de la experiencia, se ha hecho al alumno responsable y motor activo de su aprendizaje y adquisición de competencias tanto técnicas como "soft". Finalmente, se ha creado un grupo cohesionado de carácter multidisciplinar donde colaboran profesores de distintos departamentos y áreas y personal externo para la mejora de la enseñanza en competencias transversales así como la continuidad de nuevas actividades didácticas (Resultado indirecto del proyecto).

\section{UTILIDAD/ANÁLISIS}

De cara a analizar el impacto de la actividad y distintos aspectos de la misma como utilidad de la experiencia, diseño, recursos, tiempos de aprendizaje, necesidades y material de apoyo; se pasaron unas encuestas a los estudiantes de ambas asignaturas cuyos resultados se presentan a continuación. Por otro lado, como resultado de la tarea 5, indicada en la metodología, se presentan las principales limitaciones recopiladas por el equipo de trabajo sobre el proyecto ejecutado.

Cabe aclarar que la primera parte del cuestionario se dedicó a evaluar el perfil del estudiante, y a continuación de recogieron sus opiniones y valoraciones. Los resultados se presentan en las Tablas A y B del Anexo. Como puede observarse del análisis realizado, la edad media de los estudiantes está en torno a 22 años, donde el 32\% de los estudiantes eran de sexo “mujer" (M) y donde la experiencia previa fue notablemente mayor para la asignatura de Comercialización. En relación, a la autoevaluación sobre conocimientos previos, se pidió a los alumnos una valoración de 1 a 5 , donde 1 indicaba ser "muy poco hábil” y 5 "muy hábil". Los estudiantes se consideraron aceptablemente hábiles en los casos del manejo de paquetes de Ofimática, e-learning 
y redes sociales, mientras que en el caso de los mundos inmersivos y videojuegos resultaron “poco hábiles” (ver Tabla A del Anexo).

Como puede observarse de la información que aparece en la Tabla B, la valoración del entorno inmersivo (apartado A) en términos de espacios, utilidades, diseño y estilo fue considerada entre las categorías de "aceptable" y "adecuado". En relación a los recursos de apoyo, el manual fue considerado "más útil" que las propias sesiones tutoradas, lo cual ilustra el aspecto mencionado sobre la responsabilidad del estudiante como actor ejecutor del aprendizaje. Esto también queda reflejado en los resultados del apartado $C$ donde se considera la presencia de los compañeros más útil que la del tutor. La sesión de inmersión conseguida estuvo entre las categorías de "media (3)" y "alta" (4). Finalmente los tiempos de aprendizaje para el manejo básico del avatar sólo fueron documentados en la asignatura de Proyectos y fueron de alrededor de 25 minutos.

Entre las principales limitaciones detectadas habría que señalar las relacionadas con la capacidad de uso del espacio virtual diseñado de la banda que originaron problemas técnicos (bloqueo de ordenadores y ralentización de la práctica). Como recomendación final, más allá de contextualizar la actividad en un tema, sería recomendable plantear un análisis de casos más profundo que abarcara los objetivos de la asignatura.

\section{CONCLUSIONES/DISCUSIÓN}

El uso de los mundos inmersivos en casos prácticos profesionales ha resultado verdaderamente útil por dos tipos de consideraciones observadas por el presente equipo de trabajo: i) se promueve la "gamificación" de la enseñanza y el aprendizaje y se favorece la creatividad; ii) los estudiantes toman un papel activo en su aprendizaje porque asumen funciones y se familiarizan con un contexto profesional real que intentan imitar. La complementariedad y evaluación conjunta del trabajo documental entregado y la realización de los vídeos ayuda a que éstos sean considerados parte del proceso de aprendizaje y no algo meramente anecdótico o divertido. Por otro lado, la participación de los agentes externos como D. Francisco Mérida, y D. Luis Moya, fortaleció la actividad al acercar a los estudiantes a la realidad del mundo laboral y de los negocios, gracias a sus aportaciones sobre casos particulares y a los detalles sobre las situaciones cotidianas que ellos afrontan a diario y que no suelen incluirse en los libros de texto ni en las clases magistrales. Las reflexiones y comentarios de agentes externos asociados al mundo profesional constituye un complemento fundamental para acercar a los estudiantes al mundo laboral, que incentiva el aprendizaje más allá del estudio tradicional de los contenidos de las asignaturas.

\section{AgRADECIMIENTOS}

This work was partially supported by the Educative Innovation Proyect funded by the University of Cordoba. The authors would especially like to thank the students their participation and the engineer $\mathrm{M}^{\mathrm{a}}$ Carmen Beato her technical contribution to the project.

\section{BIBLIOGRAFÍA}

BAKER, S., WENTZ, R., Y WOODS, M. “Using Virtual Worlds in education: Second life as educational tools". Teaching and Psychology, 2009, 36, P 59-64.

Burdea G, COIFFeT P. "Virtual Reality Technology”. Presence: Teleoperators and virtual environments, 2003, 12/6, p. 663-664.

Redel, M. D., C. Castillo, C. Aguilar, M. J. Polo, And E. V. Taguas. "Development of a Virtual Tool for Learning Basic Organization and Planning in Rural Engineering Projects”. European Journal of Engineering Education, 2014, 39/5, p. 507-517

RODRÍGUEZ-DONAIRE S., AMANTE, B. "Collaborative environments, a way to improve quality in Higher Education". ProcediaSocial and Behavioral Sciences 2012, 46, p. $875-884$.

SCHAF F.M., MÜlLER D., BRUns F.W., PEREIRA C.E., ERBE H.-H. “Collaborative learning and engineering workspaces”. Annual Reviews in Control 2009, 33, 246-252.

SCHMORROW D.D. "Why virtual?" Theoretical Issues in Ergonomics Science 2009, 10/3, p. 279-282.

TAGUAS EV, FALCONER R, TARQUIS AM. "Engineering education on geosciences in a changing world". European Journal of Engineering Education, 2014, 39 (5),p. 463-466.

\section{ANEXO - FIGURAS Y TABLAS}

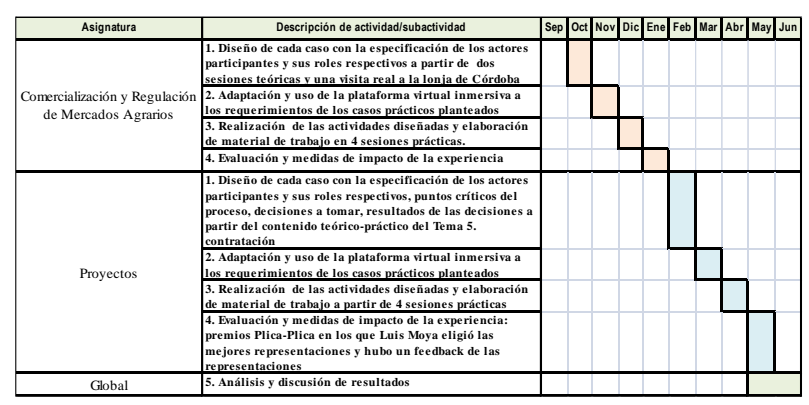


Figura 1. Cronograma de la metodología seguida y los paquetes de trabajo.

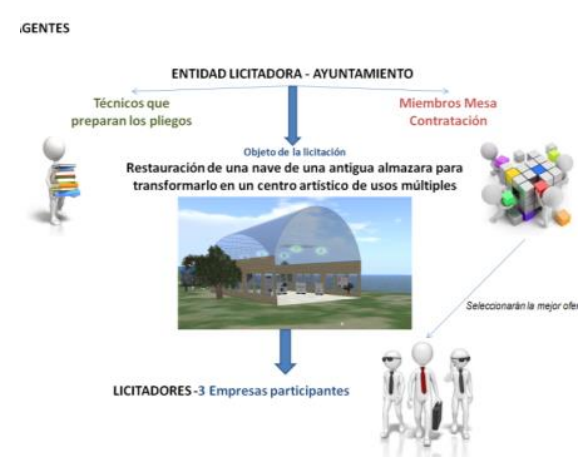

Figura 2. Agentes, objetivos y esquema introductorio para la práctica de la licitación en los mundos inmersivos.

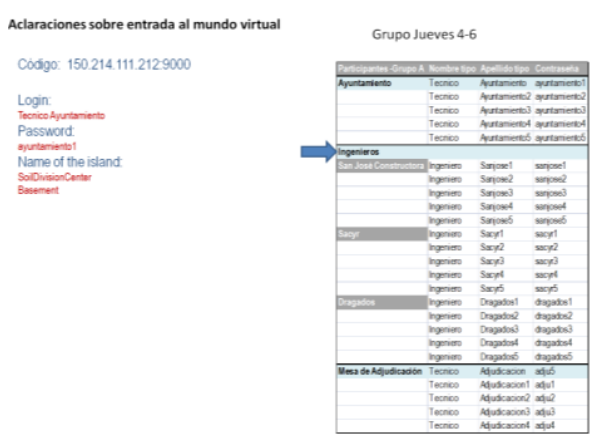

Figura 3. Datos de los usuarios de una sesión práctica para llevar a cabo la práctica.

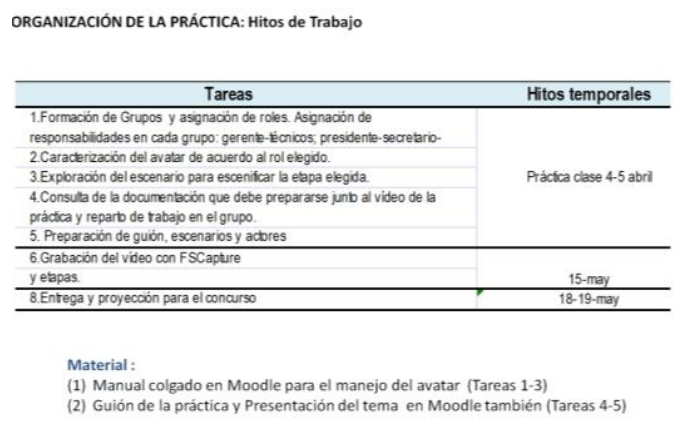

Figura 4. Programa de trabajo y material para la realización de la práctica.

Evaluación y Feedback

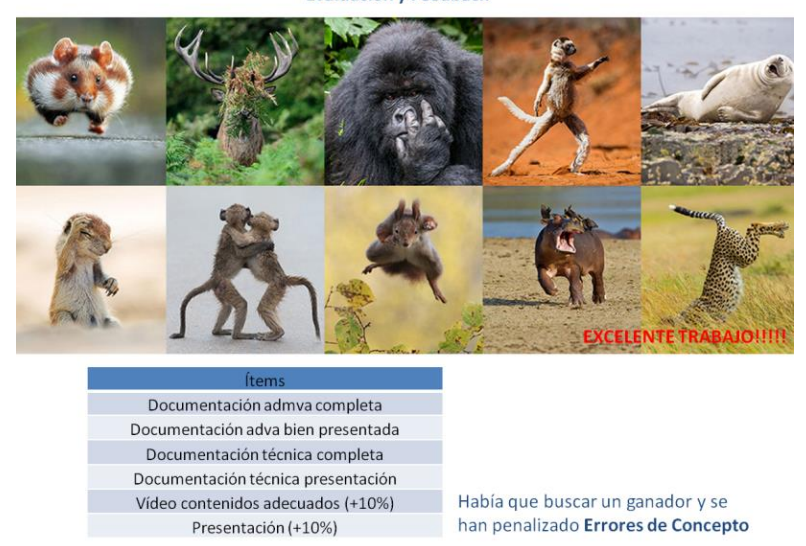


Figura 5. Ítems para la evaluación de los trabajos, y valoración general

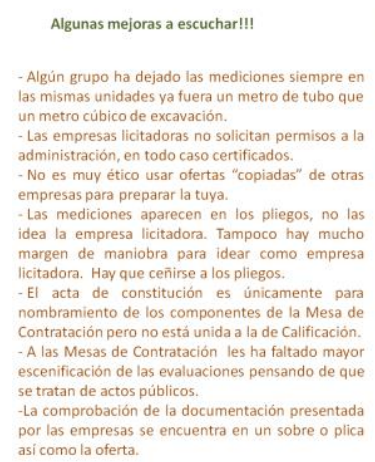

GRUPOS FINALISTAS CON RECONOCIMIENTO
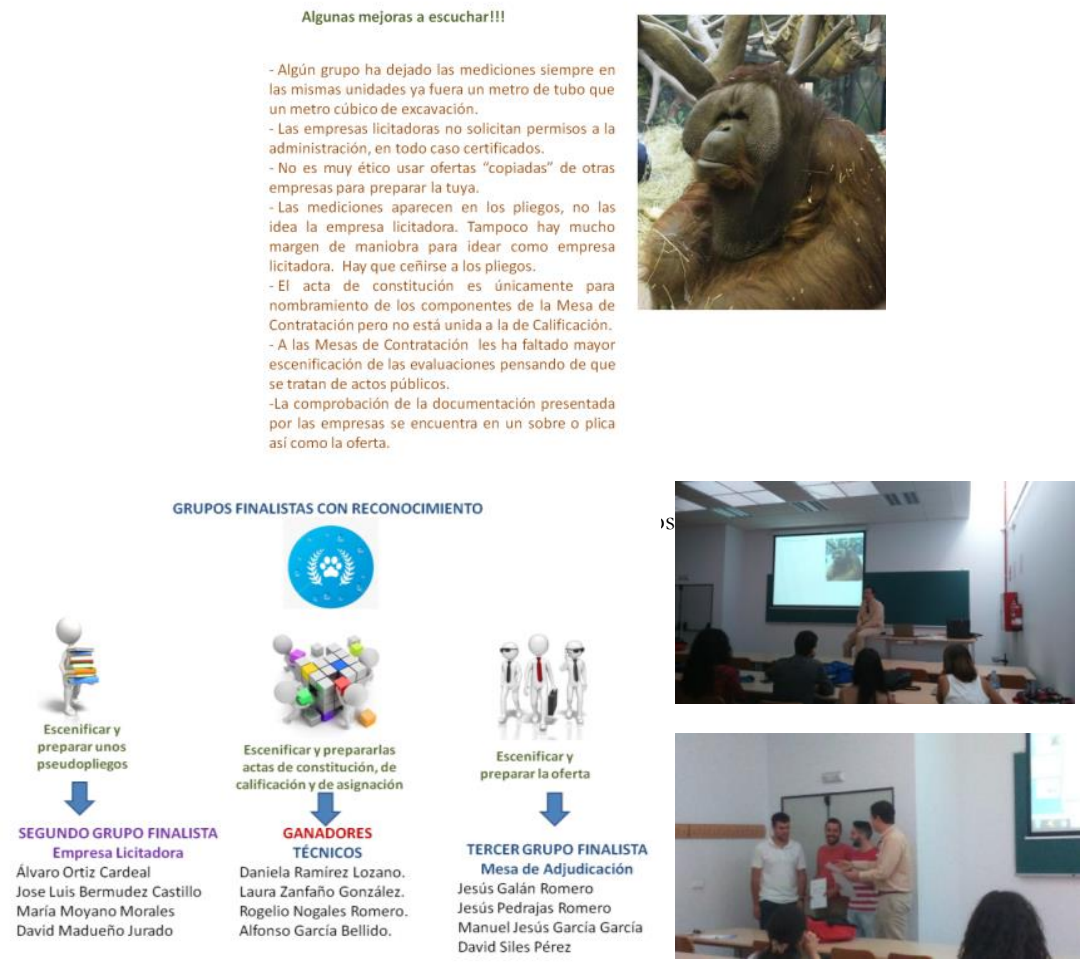

Tabla A Resumen del resultado de las encuestas de caracterización de los estudiantes. (n: tamaño muestal; M: Mujer; Escala de valoración de 1 a 5. 1:muy poco hábil, 2: poco hábil, 3: aceptable/me defiendo, 4: hábil, 5: muy hábil)

Tabla B Resumen del resultado de la valoración del espacio inmersivo y la actividad. (n: tamaño muestal; Escalas de valoración Apartado A de 1 a 5; 1:muy poco adecuado, 2: poco adecuado, 3: aceptable, 4:adecuado, 5: muy adecuado. Escalas de valoración Apartado B de 1 a 5; 1 :muy poco útil, 2: poco útil, 3: aceptable, 4:

Figura 7. Listado de los ganadores y finalistas de los premios PLICA-PLICA (a la izquierda). Foto de D. Luis Moya, Gerente de Aguas de Córdoba (arriba derecha), comentando los resultados y entregando el diploma acreditativo a los finalistas (abajo derecha).

útil, 5: muy útil. Escalas de valoración Apartado C de 1 a 5; 1:imprescindible, 2: necesaria, 3: en ocasiones , 4: en pocas ocasiones, 5: aprendizaje autónomo.

\begin{tabular}{|c|c|c|c|c|c|c|c|}
\hline \multirow{2}{*}{ Análisis preliminar } & \multirow{2}{*}{ Característica } & \multicolumn{2}{|c|}{ Proyectos $(n=19)$} & \multicolumn{2}{|c|}{ Comercialización $(n=74)$} & \multicolumn{2}{|c|}{ Total $(n=93)$} \\
\hline & & Media & Desvest & Media & Desvest & Media & Desvest \\
\hline \multirow{3}{*}{$\begin{array}{l}\text { CARACTERIZACIÓN } \\
\text { PREVIA DE LOS } \\
\text { PARTICIPANTES }\end{array}$} & EDAD & 22.4 & 2.1 & 22.1 & 2.2 & 22.2 & 2.2 \\
\hline & SEXO & $32 \% \mathrm{M}$ & - & $32 \% \mathrm{M}$ & - & $32 \% \mathrm{M}$ & \\
\hline & EXPERIENCIA PREVIA & Sí $16 \%$ & - & Sí 35\% & - & $30 \%$ Sí & \\
\hline \multirow{5}{*}{ AUTOEVALUACIÓN } & OFIMÁTICA & 3.2 & 1.0 & 3.0 & 1.0 & 3.0 & 1.0 \\
\hline & E-LEARNING & 3.2 & 1.1 & 3.4 & 1.1 & 3.4 & 1.1 \\
\hline & REDES SOCIALES & 3.2 & 1.1 & 4.0 & 1.0 & 3.8 & 1.0 \\
\hline & VIDEOJUEGOS & 2.0 & 1.2 & 3.0 & 1.6 & 2.8 & 1.5 \\
\hline & MUNDOS INMERSIVOS & 1.6 & 0.9 & 2.1 & 1.0 & 2.0 & 1.0 \\
\hline
\end{tabular}

\begin{tabular}{|c|c|c|c|c|c|c|c|}
\hline Análisis & Ítems & \multicolumn{2}{|c|}{ Proyectos $(n=19)$} & \multicolumn{2}{|c|}{ Comercialización $(n=74)$} & \multicolumn{2}{|c|}{ Total $(n=93)$} \\
\hline \multirow{3}{*}{$\begin{array}{l}\text { A. ENTORNO } \\
(1-5)\end{array}$} & Espacios & 3.2 & 0.9 & 3.4 & 0.7 & 3.4 & 0.7 \\
\hline & Utilidades & 3.0 & 1.1 & 3.5 & 0.8 & 3.4 & 0.9 \\
\hline & Diseño y estilo & 3.3 & 1.0 & 3.4 & 1.0 & 3.4 & 1.0 \\
\hline \multirow{2}{*}{$\begin{array}{l}\text { B. MANEJO } \\
\text { BÁSICO (1-5) }\end{array}$} & Sesiones tutoradas & 3.4 & 1.1 & 3.6 & 1.0 & 3.6 & 1.0 \\
\hline & Facilidad manejo & 3.3 & 1.1 & 3.6 & 0.8 & 3.5 & 0.9 \\
\hline $\begin{array}{c}\text { C. INTERACCIÓN } \\
(1-5)\end{array}$ & Presencia tutor & 2.8 & 1.3 & 2.8 & 1.2 & 2.8 & 1.3 \\
\hline \multirow{3}{*}{$\begin{array}{l}\text { D. CURVA DE } \\
\text { APRENDIZAJE } \\
\text { (Minutos) }\end{array}$} & Desplazamientos & 3.1 & 3.4 & - & - & 3.1 & 3.4 \\
\hline & Coger objetos & 5.6 & 8.6 & - & - & 5.6 & 8.6 \\
\hline & Personalizar el avatar & 16.3 & 11.3 & - & - & 16.3 & 11.3 \\
\hline
\end{tabular}

